Recent Advances in Communication, Electronics \& Electrical Engineering

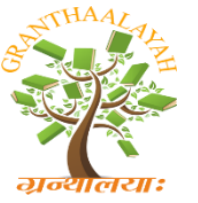

INTERNATIONAL JOURNAL OF RESEARCH -

GRANTHAALAYAH

A knowledge Repository

RACEEE - 17

\title{
WIRELESS CONTROL OF AN AUTOMOBILE USING AIR GESTURES
}

\author{
Priyamvadaa.R ${ }^{1}$, Sai Supriya.G. $K^{2}$, Savita Sangappa Mulimani ${ }^{3}$ \\ 1, 2,3 Mtech Student, Department of Digital Electronics and Communication, Dayananda Sagar \\ College of Engineering, Bangalore, India
}

DOI: https://doi.org/10.29121/granthaalayah.v5.i4RACEEE.2017.3328

\begin{abstract}
In the present world people with disabilities wish to be independent and thus the dependence on automatic machinery has increased drastically. People with physical disabilities and partial paralysis find it difficult to navigate without the assistance of someone. The system proposed will be a boost to the physically challenged people as it will help them to be self-reliable. Air gesture model is being as a key component to derive the benefit and gesture recognition is one obvious way to create a useful, highly adaptive interface between machines and their users. The gestures of the hand are read by a camera which is attached to a computer where further processing occurs. Hand gesture recognition technology allows for the navigation of an automobile using only a series of finger and hand movements, eliminating the need for physical contact between operator and machine. If the hand gesture is similar then Arduino is programmed such that the robotic car moves forward. This model can be extended to other areas such as efficient control by traffic corps, patients and senior citizens to call or interact with others, and so on. However, in this paper, the focus will be more as part of driving vehicles.
\end{abstract}

Keywords: Image Processing; Hand Gesture; Human-Machine Interface.

Cite This Article: Priyamvadaa.R, Sai Supriya.G. K, and Savita Sangappa Mulimani. (2017). "WIRELESS CONTROL OF AN AUTOMOBILE USING AIR GESTURES." International Journal of Research - Granthaalayah, 5(4) RACEEE, 79-84. https://doi.org/10.29121/granthaalayah.v5.i4RACEEE.2017.3328.

\section{Introduction}

Today's environment intent to surround the experiences to be more close to real. However, the devices of different technologies help in tracking the user and helps in identifying and moving the objects in the virtual context. Touch screens, bio-metric control based operations, voice based controls with commands could be considered as examples in existence though the touch screen is more popular. Our interest here is in formulating an alternative, natural interface that would more intimately model the way we interact with the real world. 
Recent Advances in Communication, Electronics \& Electrical Engineering

These challenges open a new direction for human-computer interaction. The computer vision devices can be implemented and upgraded to new input devices in future. It gives input command to the computer, rather than just a function of taking photo or recording video. One of the ways to give signal to computer vision devices is by way of using hand gesture. More specifically hand gesture is used as the signal or input modality to the computer. Certain signal can be recognized by computer as an input of what computer should do. These will benefits the entire user without using a direct device and can do what they want as long as the computer vision device can sense it. This makes usage of computer easier than using the keyboard or mouse. Interaction between humans comes from different sensory modes like gesture, speech, facial and body expressions. The main advantage of using hand gestures is to interact with computer as a non-contact human computer input modality. The state of the art of human computer interaction presents the facts for controlling the computer processes, using gestures of various types of hand movements. A gesture is a form of non-verbal communication or nonvocal communication in which visible bodily actions communicate particular messages, either in place of, or in conjunction with speech. Gestures include movement of the hands, face, or other parts of the body. Gestures differ from physical non-verbal communication that does not communicate specific messages, such as purely expressive displays, proxemics, or displays of joint attention. Gestures allow individuals to communicate a variety of feelings and thoughts, from contempt and hostility to approval and affection, often together with body language in addition to words when they speak. Location based identification in a context specific environment introduced, can help in extending usage of these gestures in many other areas of day to day needs. Lifesaving models can be built and put into use, using this gesture based models. Though the gesture based controlling model may be used in different areas of need, this paper is prepared with more focus on using gestures in controlling vehicles.

Hand gestures are used to control vehicle and can solve the problems while controlling vehicle and many more problems which are listed below:

There are now over 700 million motor vehicles in the world and the number is rising by more than 40 million each year. Over 1, 37,000 people were killed in road accidents in 2013 alone, that is more than the number of people killed in all our wars put together. There is one death every four minutes due to a road accident in India.[1]

There are many numbers of diseases being spread by holding the steering wheel. It becomes very difficult for the physically challenged people (person with no leg) to handle the car. One of the diseases caused by steering wheel is carpal tunnel syndrome. The carpal tunnel joint on the wrist are in close proximity as the hand grasps the steering wheel, and as a result, are vulnerable during impact. The injury can occur when the hand is braced on the steering wheel at the time of a front or rear-end collision.

In order to overcome the disadvantages and discomforts, we make use of air gesture based vehicle control technique. In this technique there is no need of steering wheel, hence one can control spreading of disease which is caused by touching the steering wheel. One will get panic during driving the car as they cannot react suddenly in a proper way. We use the above technique where the reaction time is very low since it is just controlled by a hand movement which results in reduction of accidents and diseases. 
Recent Advances in Communication, Electronics \& Electrical Engineering

\section{Materials and Methods}

\section{Materials}

Materials used (both hardware and software) are listed below. Though there are many components/elements used, some of the key are listed below.

Key Hardware Materials o Web camera

- Arduino Uno o Bluetooth

- Chassis o Wheels o Motors

- Motor Control Board (L293D) o Battery holder and Batteries o Connecting wires

- LEDs

Key Software Used

- Matlab 2011b

- Simulink

- Arduino IDE

\section{Methods}

The robot control system includes four parts:

A webcam connected with the laptop computer. A gesture recognition system running on a laptop computer, a robotic car and the robot controller. Bluetooth transmitter and receiver are connected with the gesture recognition system and the robot controller respectively.
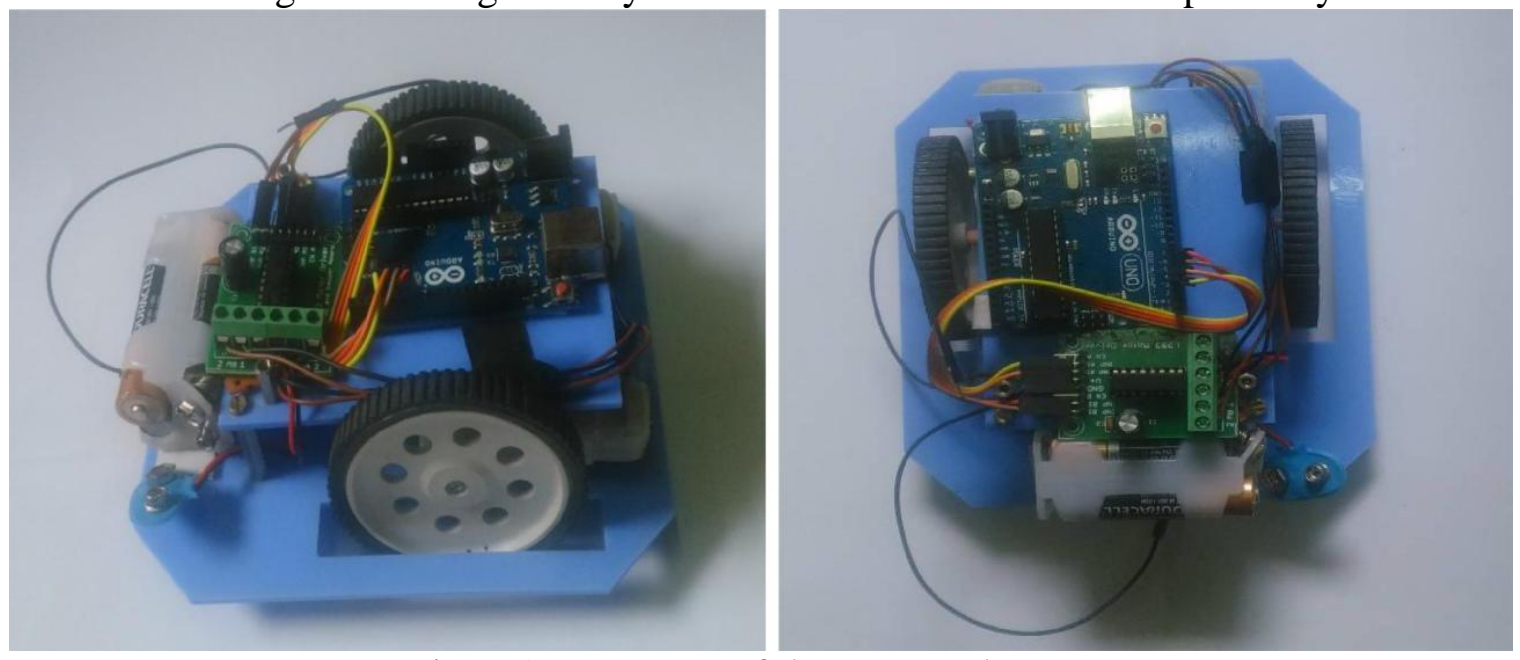

Figure1: Prototype of the proposed system

The webcam is used to obtain the image data of the human fingers. The acquired data is subjected to segmentation, erosion, dilation and identification of finger location. Then the data are processed to recognize the gesture. Each gesture corresponds to a different robot control command. The Bluetooth wireless module is used to send these different robot control commands to the robot controller. The robotic car will do actions according to different human 
Recent Advances in Communication, Electronics \& Electrical Engineering

hand gestures, thus human-robot interaction can be achieved. The gesture recognition system is developed with MATLAB.

The design and development method followed will allow users to adjust movement corresponding to the hand gesture. Gestures of hand are captured by web camera and it is sent to processor (real time gesture recognition processor) which identifies the motion of the hand in the particular area. The Gesture recognition unit comprises of a MATLAB code running on a laptop/computer. A gesture recognition system is proposed and developed which can effectively recognize hand gestures with less computing but high accuracy.

The proposed methodology for gesture recognition involves image acquisition, conversion to suitable color model, segmentation, erosion and dilation. The proposed methodology has the following process steps: first the captured image is converted into suitable color model. Suitable color model is selected by analyzing the captured image. Segmentation of the images follows the analyzing of the images. The main purpose of segmentation is to simplify or change the representation of an image into something that is more meaningful and easier to analyze. Erosion is carried out after the segmentation, to remove the noise, if any, generated during segmentation. Information lost, if any, during erosion is received as part of Dilation. After all these processes, the finger location is identified by using its coordinates. Once a hand gesture is recognized, an appropriate command is sent to a robot. After the robot receives a command, it performs a predefined work and keeps repeating until a new command arrives. Commands for movement are written as a function using the coordinates through Matlab. We define gestures to direct the operation of the robot. The operations includes the following movements: "Straight", "Reverse", "Left", "Right" and "Stop". There are other indicators such as "Left indicator", "Right Indicator", "Horn", which can also be used using predefined number indicating gestures. The experimental results are very encouraging as the system produces real-time responses and highly accurate recognition towards various gestures.

\section{Results and Discussions}

First we built a robot, which could just go straight, and then we programmed it to accept serial inputs from keyboard. We could move the automobile as desired by us. Once we got the movements of the car, we shifted back to SIMULINK for further coding. We further enhanced the processing environment, did various trial and error methods to find out the threshold values at different lighting conditions. We finally found values adjusting to all lighting conditions and most of the hands. We also added indicators and horn to the model.

We attached the output of processing to COM port which was connected to the Arduino board. As soon as the gestures were detected, the car would move in the desired direction.

Discussion started with usage of latest and cost effective devices and addressing the challenges which we identified.

The working model enabled a point of discussion of, how this can be extended in certain other areas such as

- Gesture based traffic control 
Recent Advances in Communication, Electronics \& Electrical Engineering

- Convenience provided to a senior citizens on their mobility and addressing their needs

- Patients in the hospital using the gestures to call the doctor or help when needed and so on.

- Cars deviating or moving out of the assigned lanes to be directed towards the correct lane. (gesture signals to move to the right or left to follow the correct lane discipline)

\section{Conclusions \& Recommendations}

We have created a new algorithm, for hand gesture recognition which in-turn will control a robot. We have measured the effectiveness of the algorithm on real images we have acquired for efficiency. Reliable performance of hand gesture recognition techniques in a general setting require dealing with occlusions, temporal tracking for recognizing dynamic gestures, as well as $3 \mathrm{D}$ modeling of the hand, which are still mostly beyond the current state of the art.

Our system has shown the possibility that interaction with machines through gestures is a feasible task. These gestures can be extended to vehicles on road in addition to controlling in a limited environment. More tasks can be included as part of controlling with variety of formats of gestures, which are programmed with commands.

New technology is enabling automobile manufacturers to integrate gesture recognition features in their cars to let drivers manage the control systems of the car. For example, an approaching hand can activate the in-car infotainment system, or tilting your head can switch on the turning indicator.

Controlling a robot, in real time, through the hand gestures is a novel approach. The approach has huge potential once it gets further optimized. Use of more efficient wireless communication technique and a camera on the robot unit would improve the performance of system to a great extent and can be incorporated in future work.

The technology is new and in its infancy today. The rapid pace of innovation and its ease of use will shift the buyers towards favoring a car with gesture recognition system in the future. As the technology concerning gesture-based and speech-based control systems improves, more features could be added to the car. Not only could hand and leg movements be detected, but a slight head movement could also be mapped to perform a certain function.

There are challenges associated with the accuracy and usefulness of gesture recognition software due to the limitations on the equipment used and image noise. Images or video may not be under consistent lighting, or in the same location. Items in the background or distinct features of the users may make recognition more difficult. The variety of implementations for image-based gesture recognition may also cause issue for viability of the technology to general usage. For example, an algorithm calibrated for one camera may not work for a different camera.

In order to capture human gestures by visual sensors, robust computer vision methods are required. Since some of the major concerns are identified during the initial phase itself, it can be corrected with latest technology and the enhancements which are being available for use. The potential for gesture technology is immense and it will only improve as we move into the future. 
Recent Advances in Communication, Electronics \& Electrical Engineering

\section{Acknowledgement}

We would like to thank Mr.Krishnananda, Assistant Professor, Dayananda Sagar College of Engineering, Bangalore for his inputs and guidance.

\section{References}

[1] https://www.ielts.org/-/media/pdfs/115013_academic_reading_sample_task__matching_information__2_.ashx?la=en

[2] Haseru Chen, Yohei Kakiuchi, Manabu Saito, Kei Okada, Masayuki Inaba "VIEW-BASED MULTI-TOUCH GESTURE INTERFACE FOR FURNITURE MANIPULATION ROBOTS".

[3] Kazuma Yoshino, Hiroyuki Shinoda "ACOUSTIC SCREEN FOR VISIO CONTACT LESS SENSATION TOUCH INTERFACE WITH TACTILE".

[4] Radovan Stojanović, Nedjeljko Lekić, Zoran Mijanović, and Jovan Kovačević "FPGA BASED CAPACITIVE TOUCH PAD/INTERFACE ."

[5] Liang Zhang,Jigao Fu,Zhubing Wang,Chen MaBo Chen Pingfan He "THE DESIGN OF TOUCH-SCREEN WITH HIGH RESOLUTION INFRARED BASED ON PLASTIC OPTICAL FIBER AND IMAGE PROCESSING".

[6] Yu-Hsuan Wang, , Xiao Li , Chen Zhao, and Xinyu Liu "A PAPER-BASED PIEZOELECTRIC TOUCH PAD INTEGRATING ZINC OXIDE NANOWIRES”.

*Corresponding author.

E-mail address: rpriyamvadaa@gmail.com 